\section{Kidney \\ Blood Pressure \\ Research}

\title{
Influence of L-Carnitine Supplementation on Serum Lipid Profile in Hemodialysis Patients: A Systematic Review and Meta- Analysis
}

\author{
Haohai Huang ${ }^{a}$ Lijun Song ${ }^{a}$ Hua Zhang ${ }^{b}$ Hanbin Zhang ${ }^{b}$ Jiping Zhang ${ }^{c}$ \\ Wenchang Zhao ${ }^{a}$
}

aSchool of Pharmacy, Guangdong Medical College, Dongguan; 'bino-American Cancer Research Institute, Key Laboratory for Medical Molecular Diagnostics of Guangdong Province, Guangdong Medical College, Dongguan; 'Department of Science and Education, The Second People's Hospital of Foshan, Foshan, Guangdong, China

\section{Key Words}

Hemodialysis $\bullet$ L-Carnitine $\bullet$ Meta-Analysis

\begin{abstract}
Background/Aims: An increasing body of evidence demonstrates that L-carnitine plays a pivotal role in lipid metabolism of hemodialysis (HD) patients. However, there are still some reservations about its benefits. Therefore, we performed a meta-analysis to assess the effects of L-carnitine supplementation on lipid profile in HD patients. Methods: Literature search was performed to identify the relevant randomized controlled trials that investigated the effects of L-carnitine on the lipid profile of subjects. Two independent authors used an Excel file to extract data and assess trials quality. The primary effect measure was the difference in means of the final lipid measurements between the intervention and control groups. The metaanalysis was performed with the fixed-effects model or random-effects model according to heterogeneity. Results: Twelve studies with a total of 391 patients met the inclusion criteria. The use of $\mathrm{L}$-carnitine was not associated with a reduction in the total cholesterol $(S M D,-0.11$; $95 \% \mathrm{CI},-0.31$ to 0.09$)$, HDL-cholesterol (SMD, $0.01 ; 95 \% \mathrm{CI},-0.36$ to 0.39), VLDL-cholesterol (SMD, 0.54; 95\% CI, -0.06 to 1.14), and the serum triglycerides (SMD, $-0.12 ; 95 \% \mathrm{CI},-0.36$ to 0.12 ). However, L-carnitine can significantly decrease the LDL-cholesterol (SMD, $-0.29 ; 95 \%$ $\mathrm{CI},-0.53$ to -0.06 ) in $\mathrm{HD}$ patients. In a subgroup meta-analysis, a significant LDL-cholesterollowering effect of L-carnitine supplementation was observed in intravenous application group, and patients with longer interventional duration and renal diseases. Conclusion: The limited evidence suggests that there was no effect of L-carnitine on serum total cholesterol, HDLcholesterol, VLDL-cholesterol and serum triglycerides. By contrast, this meta-analysis suggests a promising effect of L-carnitine on LDL-cholesterol. Further large-scale, well-designed randomized controlled trials are urgently needed.
\end{abstract}




\section{Kidney Blood Pressure Research}

Huang/Song/Zhang/Zhang/Zhang/Zhao: L-carnitine Supplementation in HD Patients

\section{Introduction}

Chronic kidney disease (CKD), especially end-stage renal disease (ESRD), is the most frequently health problem and main cause leading to kidney-related deaths. It currently affects approximately 40.3 million adults in 2010 , and is projected to reach 54.8 million in 2020 [1]. At present, kidney replacement therapy is the main measure to treat kidney failure, which dramatically improved patients' survival and the quality of life. It has been reported that a number of factors and co-morbid conditions common in dialysis are implicated as risk factors, including diabetes, hypertension, hyperlipidemia, inflammation, anemia and imbalances in mineral metabolism [2]. Among them, one of the most important complications of cardiovascular origin and the risk of cardiovascular disease in patients with chronic renal disease is hyperlipidemias [3]. It is well known that the concentration of serum lipid is a high burden of patients with dyslipidemia and cardiovascular disease, while cholesterol and triglycerides are objective index for serum lipid, therefore, reduction of cholesterol and triglycerides is important to cut down the overall mortality and mortality in HD patients.

L-carnitine, a small compound, is found mostly in milk and meat. Studies also showed that liver can synthesize the endogenous carnitine from lysine, methionine and ascorbate, niacin, pyridoxine, and $\mathrm{Fe}^{2+}[4]$. L-carnitine plays an important role in the beta-oxidation of fatty acids and can cut down free fatty acid availability for triglyceride synthesis [5, 6]. A number of studies have demonstrated that administration of L-carnitine and its analogues acetyl and propionyl L-carnitine was effective for normalize the concentrations of carnitine, plasma cholesterol, triglycerides in a streptozocin-induced diabetic rat model [7]. Further research has demonstrated that treatment with L-carnitine could prevent the progression of atherosclerotic lesions in hypercholesterolemic rabbit because of its antioxidant and lipidlowering effects [8]. Recently, a great number of randomized, placebo-controlled studies regarding the effect of L-carnitine supplementation have been reported in patients who undergoing HD. However, these studies have a modest sample size and convey inconclusive results. To better understand the efficacy of L-carnitine supplementation on serum lipid profile in HD patients, we therefore carried out a comprehensive systematic review and metaanalysis of randomized controlled trials to assess the lipid-lowering effects of L-carnitine supplementation in HD patients.

\section{Materials and Methods}

\section{Literature search and inclusion criteria}

Following the PRISMA statement guidelines for meta-analysis of randomized controlled trials (RCTs) [9], relevant RCTs were identified by searching PubMed and Embase databases (up to July 2013). We also searched The Cochrane Central Register of Controlled Trials (CENTRAL) (also up to July 2013). The structured search strategies used the following format of search terms: ("vitamin BT" OR L-carnitine OR "L carnitine" OR levocarnitine OR bicarnesine OR L-acetylcarnitine OR acetyl-L-carnitine) AND (hemodialysis OR dialysis OR "kidney replacement therapy"). Results were limited to human subjects and English-language publications. The abstracts and meeting proceedings are not included. In addition, we also searched for any additional studies in the reference lists of recent meta-analyses of L-carnitine treatment for HD patients. This process was performed iteratively until no additional articles could be identified.

The full-text publications should be included when the following inclusion criteria were met: (a) study design, RCT reported in a full paper article; (b) study population, adult patients receving HD; (c) intervention, L-carnitine supplement (no matter what type and regimen applied); (d) comparison intervention, placebo or no intervention; and (e)outcome measure, articles reported 'baseline' and 'end of intervention' mean and standard deviation values of lipid measurements for the active (intervention) and control groups.

Trials were excluded if they (a) were non-randomized studies or cross-over study design (b) were abstracts, letters, or meeting proceedings; (c) had repeated data, insufficient data or did not report outcomes of interest; and (d) enrolled patients were younger than age 18 years or patients receving peritoneal dialysis (PD). 


\section{Kidney \\ Blood Pressure Research}

\section{Data extraction and quality assessment}

Two reviewers (H.H. and L.S.) independently assessed articles for inclusion and tabulated the data from each RCT. The following information was extracted from the eligible studies: first author, year of publication, number of patients (intervention/control), mean age, gender, duration of treatment, types of L-carnitine, regimens of L-carnitine supplementation (dosage, timing), location and time on HD. We also extracted information on the baseline and final concentrations (or net changes) of serum total cholesterol (TC), LDL cholesterol, HDL cholesterol, VLDL cholesterol and triglycerides (TG). Studies that reported results in $\mathrm{mmol} / \mathrm{l}$ were converted to $\mathrm{mg} / \mathrm{dl}$ using the standard conversion factors (which was a division of the mmol/l value by 0.02586 for TC, LDL and HDL; and by 0.01129 for TG). Extracted data were entered into a standardized Excel file and were checked by another author (W.Z.). It should be emphasized that if the same population was reported in several publications, we only retained the most informative article or complete study to avoid duplication of information. Discrepancies in the evaluation of some of the studies were resolved through discussion between the authors.

Methodological quality evaluation was independently performed by two of the authors (HHH and LJS) using validated Jadad five-point scale [10]. This tool places emphasis on the following three areas when defining the quality of an RCT: (a) randomization (0-2), (b) double-blinding (0-2), and (c) description of withdrawals and drop-outs (0-1). Jadad scores ranged from 0 to 5 , when the score $\leq 2$ indicates the lowest quality and $\geq 3$ means the highest quality [11]. Disagreements were resolved through discussion and consensus.

\section{Statistical analysis}

All outcome measures were expressed as standardized mean differences (SMDs) with 95\% confidence intervals (CIs) using either fixed or random-effects model depending on the amount of heterogeneity. A random-effects model meta-analysis will be conducted for heterogeneous outcomes and a fixed-effect model meta-analysis for homogeneous outcomes. Heterogeneity across studies was tested by using the $I^{2}$ statistic (percentage of the variability in effect estimates that is due to heterogeneity rather than sampling error), and the $I^{2}$ can be calculated as: $I^{2}=100 \% \times(Q-d f) / Q(Q=$ Cochrane's heterogeneity statistics, $d f=$ degrees of freedom), its ranges between 0 and $100 \%$. Studies with an $I^{2}$ statistic of $25-50 \%$ are considered to have low heterogeneity, those with an $I^{2}$ statistic of $50-75 \%$ are referred to as moderate estimates, and those with an $I^{2}$ statistic of $>75 \%$ are referred to as high heterogeneity [12]. Subgroup analysis were planned a priori to determine whether the types of interventions (orally supplementation compared with intravenous application), interventional duration (shorter term compared with longer term), location of subject population (Asian, Europe, and America), or diseases status modified the effect of L-carnitine on lipid profile or explained any heterogeneity seen. We further conducted sensitivity analyses to explore possible explanations for heterogeneity and to examine the influence of various exclusion criteria on the overall pooled estimate. We also investigated the influence of a single study on the overall pooled estimate by omitting one study in each turn. A $p$ value $<0.05$ was judged as statistically significant. We assessed the presence of publication bias by examining funnel plot [13]. All analyses were undertaken using Review Manager (Version 5.1.Copenhagen: The Nordic Cochrane Centre, The Cochrane Collaboration, 2011).

\section{Results}

\section{Study identification and selection}

The electronic database (PubMed, Embase, CENTRAL) were searched to identify the potential studies, respectively. A total of 58 citations were obtained by the initial database search. Ten RCTs were excluded because of duplicate studies, and 31 RCTs were excluded after we had reviewed titles and abstracts, mainly because they were reviews, letter, meeting proceedings, or not relevant to our analysis. Moreover, according to the inclusion and exclusion criteria, eight articles were further excluded for the reasons as follows: one study was crossover trial, one study with unavailable data for analysis, and six studies did not report the interest outcomes. Finally, 12 RCTs that met our inclusion criteria were included in the present meta-analysis [14-25], of which three studies were determined through checking reference 


\section{Kidney Blood Pressure Research}

lists of retrieved articles [16-18]. The details of study selection flow were explicitly described in Figure 1.

Characteristics of eligible studies

The main characteristics of the 12 RCTs included in the meta-analysis are summarizes in Table 1. These studies were published from 1980 to 2012. A total of 391 patients $(209$ in the L-carnitine group and 182 in control group) were included in this analysis. All trials included both men and women. Of these 12 included studies, 9 studies compared L-carnitine to placebo [14-21, 25] while

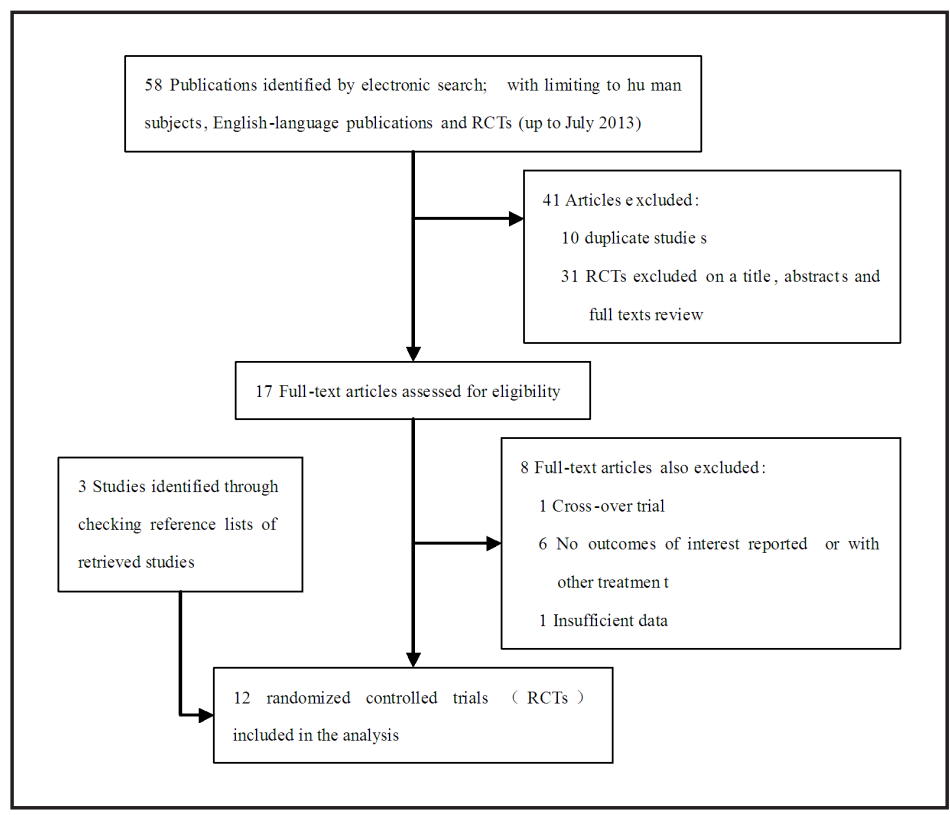

Fig. 1. Flow of studies through the review. RCTs: randomized controlled trials. 3 studies compared to no treatment [22-24]. The types of interventions were oral or given intravenously. The total dosage of L-carnitine supplementation in the intervention groups ranged from 0.5 to $1 \mathrm{~g}$. The treatment duration ranged from 5 weeks to 24 weeks. Among the 12 studies included in the meta-analysis, 7 articles included patients with end-stage renal disease (ESRD) [1822, 24, 25], 2 included patients with chronically uremic [14, 15], 2 included patients with hyperlipoproteinaemia $[16,17]$ and 1 included patients with renal failure [23]. These include articles are all reported the change of total serum cholesterol, whereas HDL-cholesterol changes were available from 8 reports [15-19, 21, 24, 25], LDL-cholesterol from 8 studies $[15-18,21,22,24,25]$, and VLDL-cholesterol in only 2 trials [15, 18]. Serum triglycerides variations were analyzed from 10 studies [14-17, 19-22, 24, 25]. We assessed the quality of the included articles using the Jadad quality score, the Jadad score for each article was graded from scores 2 to 5 in this analysis (Table 2). Funnel plots suggested no significant asymmetry in the meta-analyses of TC, LDL-cholesterol, TG and HDL-cholesterol. Thus, there was no publication bias amongst the 12 trials (Figures not shown).

Analysis of the effects of L-carnitine supplementation on total serum cholesterol, $H D L$ cholesterol, LDL-cholesterol and VLDL-cholesterol

All 12 articles reported the changes of total serum cholesterol in study patients. The aggregated results of these studies suggest that the total serum cholesterol was not significant decreased in the L-carnitine treatment group (SMD, -0.11; 95\% CI, -0.31 to 0.09; Figure 2). There was no evidence of heterogeneity for the outcomes $\left(I^{2}=0 \%\right)$. The standardized mean difference $(95 \% \mathrm{CI})$ of the changes of HDL-cholesterol for patients treated with L-carnitine compared with control was $0.01(-0.36$ to 0.39$)$, which was not statistical significance. (Figure 3). Heterogeneity was noted for this outcome $\left(I^{2}=55 \%\right)$. The effect of L-carnitine on LDL-cholesterol was assessed in 8 trials [15-18, 21, 22, 24, 25]. Based on the results of metaanalysis, the use of L-carnitine can significantly decrease the LDL-cholesterol level (SMD, -0.29 ; $95 \%$ CI, -0.53 to -0.06 ; Figure 4 ). Heterogeneity was insignificant for this outcome $\left(I^{2}=0 \%\right)$. Only 2 articles reported the effect of L-carnitine on VLDL-cholesterol level [15, $18]$, the standardized mean difference $(95 \% \mathrm{CI})$ of the variations of VLDL-cholesterol for patients treated with L-carnitine compared with control was 0.54 (-0.06 to 1.14 ), which was 


\section{Kidney \\ Blood Pressure Research}

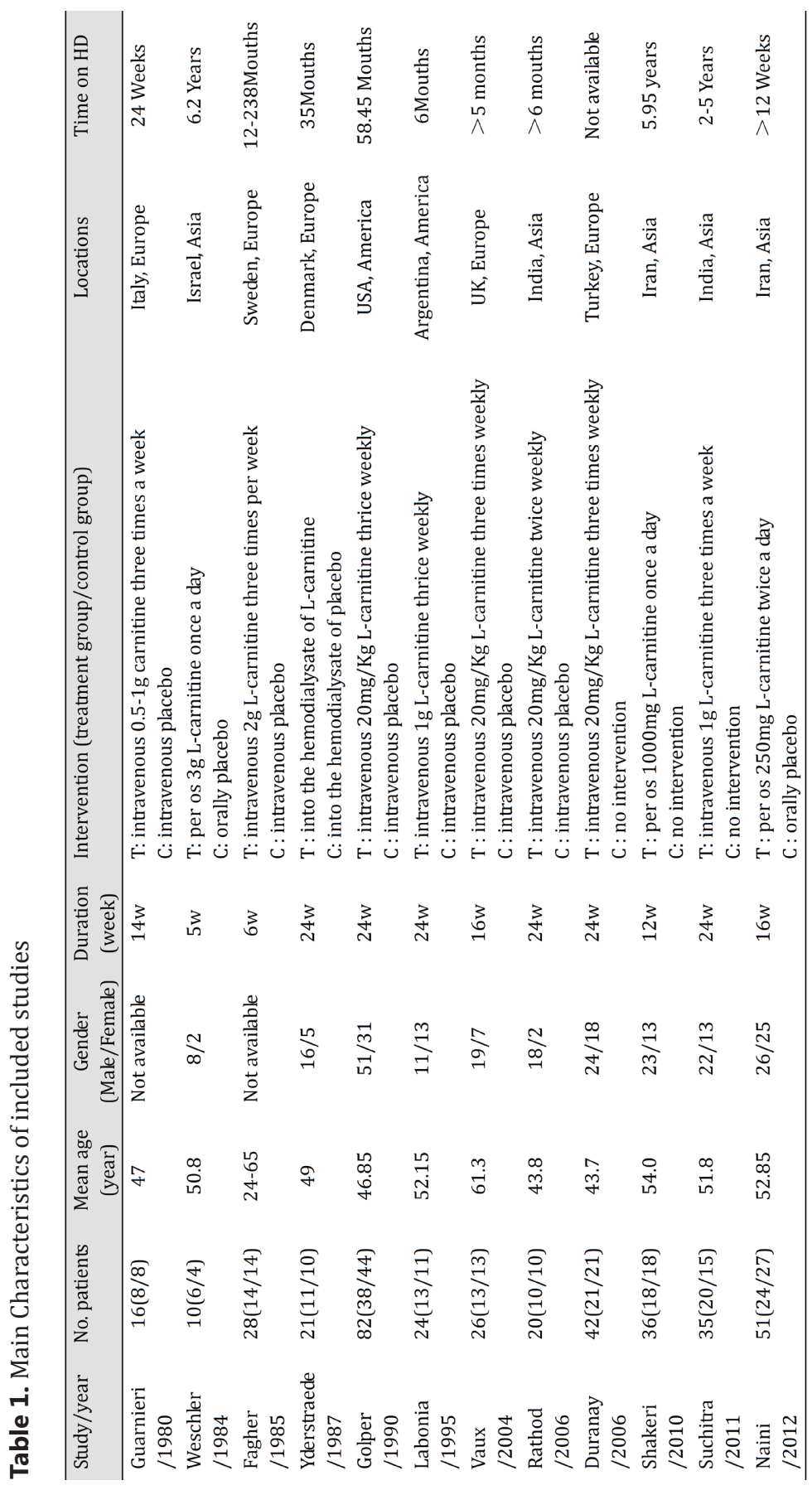

not statistical significance (Figure 5). Minimal heterogeneity was noted for this outcome $\left(I^{2}=22 \%\right)$.

Analysis of the effect of L-carnitine supplementation on serum triglycerides

Ten included studies reported changes in triglycerides [14-17, 1922, 24, 25], L-carnitine supplementation was not associated with a significant decrease in serum triglycerides level compared with that of controls (SMD, $-0.12 ; 95 \%$ CI, -0.36 to 0.12 ; Figure 6). The test for heterogeneity was insignificant $\left(I^{2}=0 \%\right)$.

Subgroup analysis and sensitivity analyses

Subgroup analysis was carried out with the data segregated by types of interventions, interventional duration, locations and diseases status of participants (Table 3). Trials with short duration $(\leq 16$ weeks) showed a non-significant reduction in TC, LDL, HDL and TG levels. Studies with longer duration (>16weeks) showed a significant reduction in serum LDL (SMD, -0.36; 95\%CI, -0.64 to -0.08 ), but a non-significant reduction in serum TC, HDL and

TG levels. Subgroup analyses according to the types of interventions showed that orally supplementation could not significantly reduce TC, LDL, HDL and TG in participants. Trials of the intravenous application group showed a significant reduction in serum LDL (SMD, $-0.31 ; 95 \%$ CI, -0.58 to -0.05 ), but a non-significant reduction in serum TC, HDL and TG levels. Analysis by diseases status in which study was carried out revealed a significant reduction in serum LDL (SMD, -0.31 ; $95 \%$ CI, -0.57 to -0.05 ) for the patients with CKD. In addition, Analysis by location in which study was carried out revealed a non-significant reduction in TC, LDL, HDL and TG levels in Asia, Europe and America population.

Subsequently, sensitivity analysis was performed by omitting the studies with low quality (Jadad score $<3$ ), the pooled results did not change substantially. When we excluded 


\section{Kidney \\ Blood Pressure Research}

Kidney Blood Press Res 2013;38:31-41

DOI: 10.1159/000355751

Published onlıne: February 06, 2014

C 2014 S. Karger AG, Base

www.karger.com/kbr

Table 2. Quality assessment of the included studies (Jadad score)

\begin{tabular}{lcccccc}
\hline Study/year & $\begin{array}{c}\text { Described } \\
\text { as randomized }\end{array}$ & $\begin{array}{c}\text { appropriate } \\
\text { randomiza- } \\
\text { tion }\end{array}$ & $\begin{array}{c}\text { Blinded } \\
\text { to } \\
\text { intervention }\end{array}$ & $\begin{array}{c}\text { Appropriate } \\
\text { blinded }\end{array}$ & $\begin{array}{c}\text { Withdrawals } \\
\text { and dropouts }\end{array}$ & $\begin{array}{c}\text { Sum } \\
\text { (Jadad } \\
\text { score) }\end{array}$ \\
\hline Guarnieri /1980 & 1 & 0 & 0 & 0 & 1 & 2 \\
Weschler /1984 & 1 & 0 & 0 & 0 & 1 & 2 \\
Fagher /1985 & 0 & 0 & 1 & 1 & 1 & 3 \\
Yderstraede /1987 & 0 & 0 & 1 & 1 & 1 & 3 \\
Golper /1990 & 1 & 1 & 1 & 1 & 1 & 5 \\
Labonia /1995 & 1 & 1 & 1 & 1 & 1 & 5 \\
Vaux /2004 & 1 & 0 & 1 & 1 & 1 & 4 \\
Rathod /2006 & 1 & 1 & 0 & 0 & 1 & 3 \\
Duranay /2006 & 1 & 1 & 0 & 0 & 1 & 3 \\
Shakeri /2010 & 1 & 0 & 0 & 0 & 1 & 2 \\
Suchitra /2011 & 1 & 0 & 0 & 0 & 1 & 2 \\
Emami Naini/2012 & 1 & 1 & 1 & 1 & 1 & 5 \\
\hline
\end{tabular}

Points were awarded as follows: study described as randomized, one point; appropriate randomization, one point, subjects blinded to intervention, one point; evaluator blinded to intervention, one point; description of withdrawals and dropouts, one point.

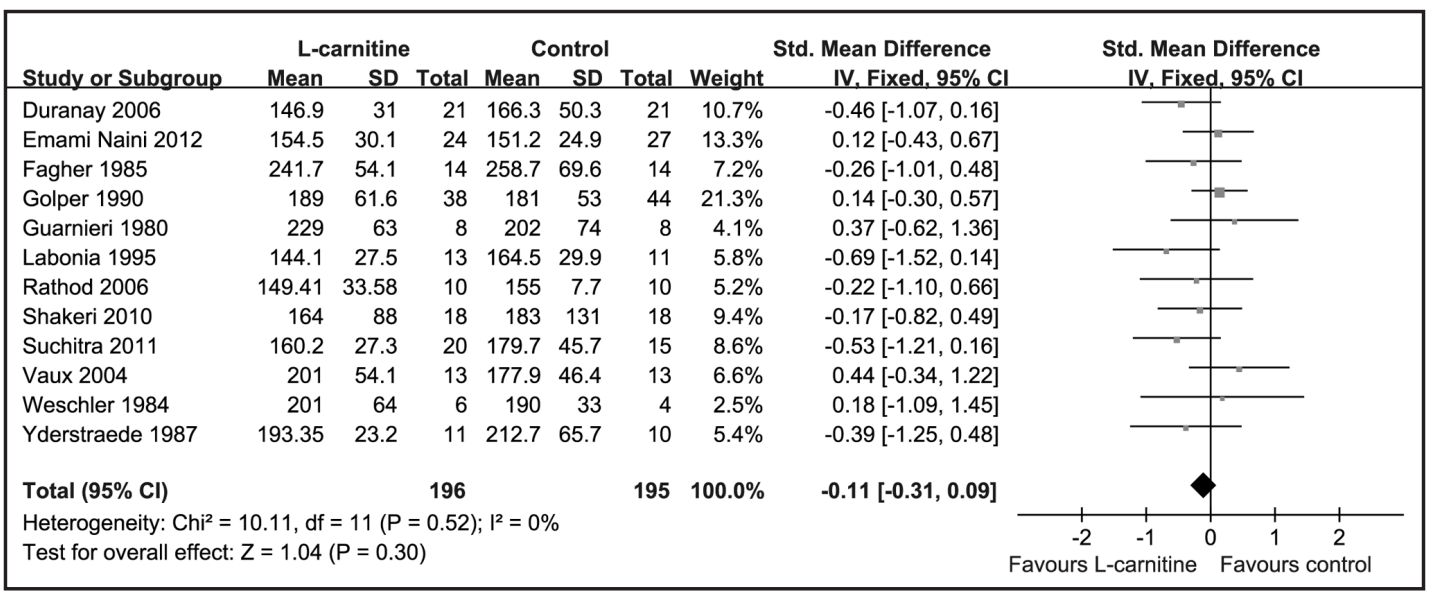

Fig. 2. Forest plot of studies comparing the effect of L-carnitine versus control on total serum cholesterol in HD patients. The sizes of the data markers indicate the weight of each study in the analysis. IV, inverse variance; fixed, fixed effects model. Values are in $\mathrm{mg} / \mathrm{dl}$.

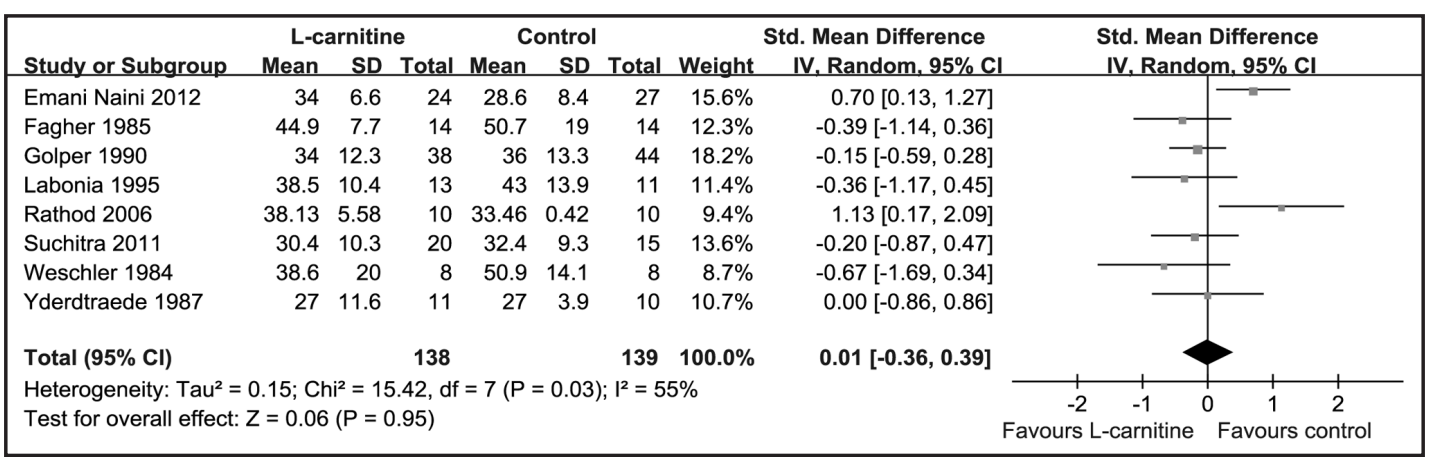

Fig. 3. Forest plot of studies comparing the effect of L-carnitine versus control on HDL-cholesterol in HD patients. The sizes of the data markers indicate the weight of each study in the analysis. IV, inverse variance; random, random effects model. Values are in $\mathrm{mg} / \mathrm{dl}$.

the two studies (Rathod et al. and Fagher et al.), our findings were similar without great fluctuation (Table 3). Further exclusion were conducted by omitting any single study did not materially alter the overall combined SMD (data not shown), which adds robustness to our main finding. 


\section{Kidney \\ Blood Pressure Research}

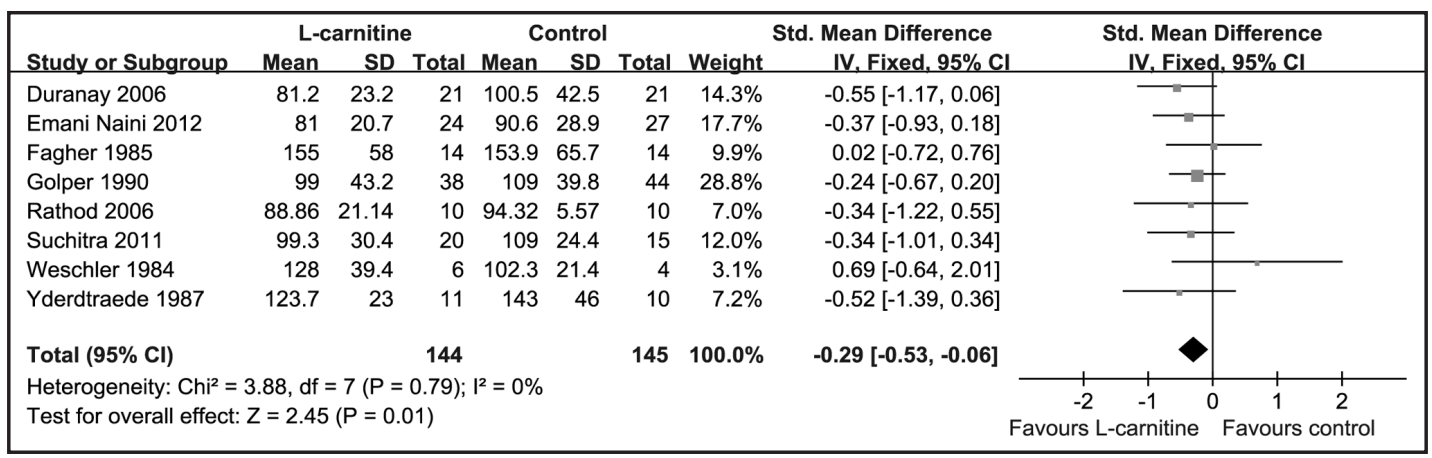

Fig. 4. Forest plot of studies comparing the effect of L-carnitine versus control on LDL-cholesterol in HD patients. The sizes of the data markers indicate the weight of each study in the analysis. IV, inverse variance; fixed, fixed effects model. Values are in $\mathrm{mg} / \mathrm{dl}$.

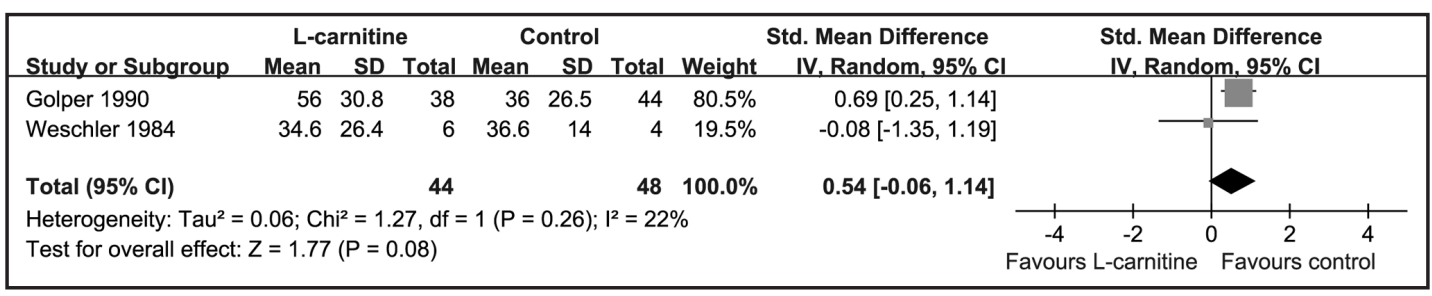

Fig. 5. Forest plot of studies comparing the effect of L-carnitine versus control on VLDL-cholesterol in HD patients. The sizes of the data markers indicate the weight of each study in the analysis. IV, inverse variance; random, random effects model. Values are in $\mathrm{mg} / \mathrm{dl}$.

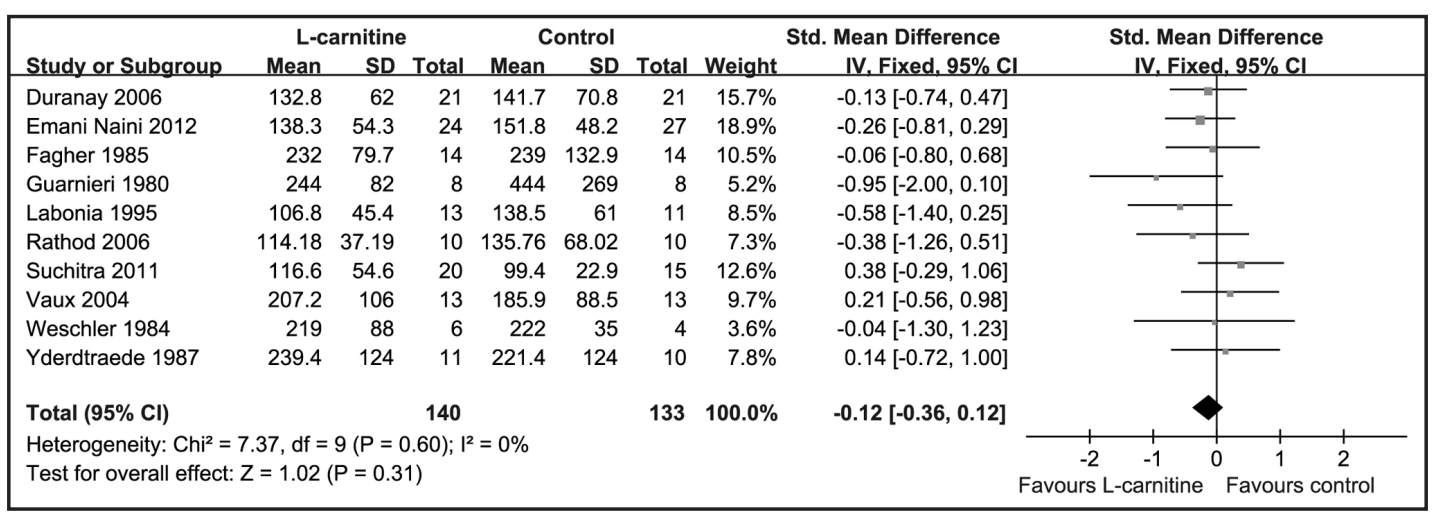

Fig. 6. Forest plot of studies comparing the effect of L-carnitine versus control on serum triglycerides in HD patients. The sizes of the data markers indicate the weight of each study in the analysis. IV, inverse variance; fixed, fixed effects model. Values are in mg/dl.

\section{Discussion}

This is a further systematic review and meta-analysis to evaluate the effects of L-carnitine supplementation on the serum lipid level. The present meta-analysis of 12 randomized controlled trials showed that L-carnitine supplementation was not associated with a significant decrease in total serum cholesterol, HDL-cholesterol, VLDL-cholesterol and serum triglycerides level. However, L-carnitine supplementation could significantly decrease the LDL-cholesterol level in HD patients. 


\section{Kidney \\ Blood Pressure Research}

Kidney Blood Press Res 2013;38:31-41

DOI: 10.1159/000355751
Published onlıne: February 06, 2014

(C) 2014 S. Karger AG, Basel

www.karger.com/kbr

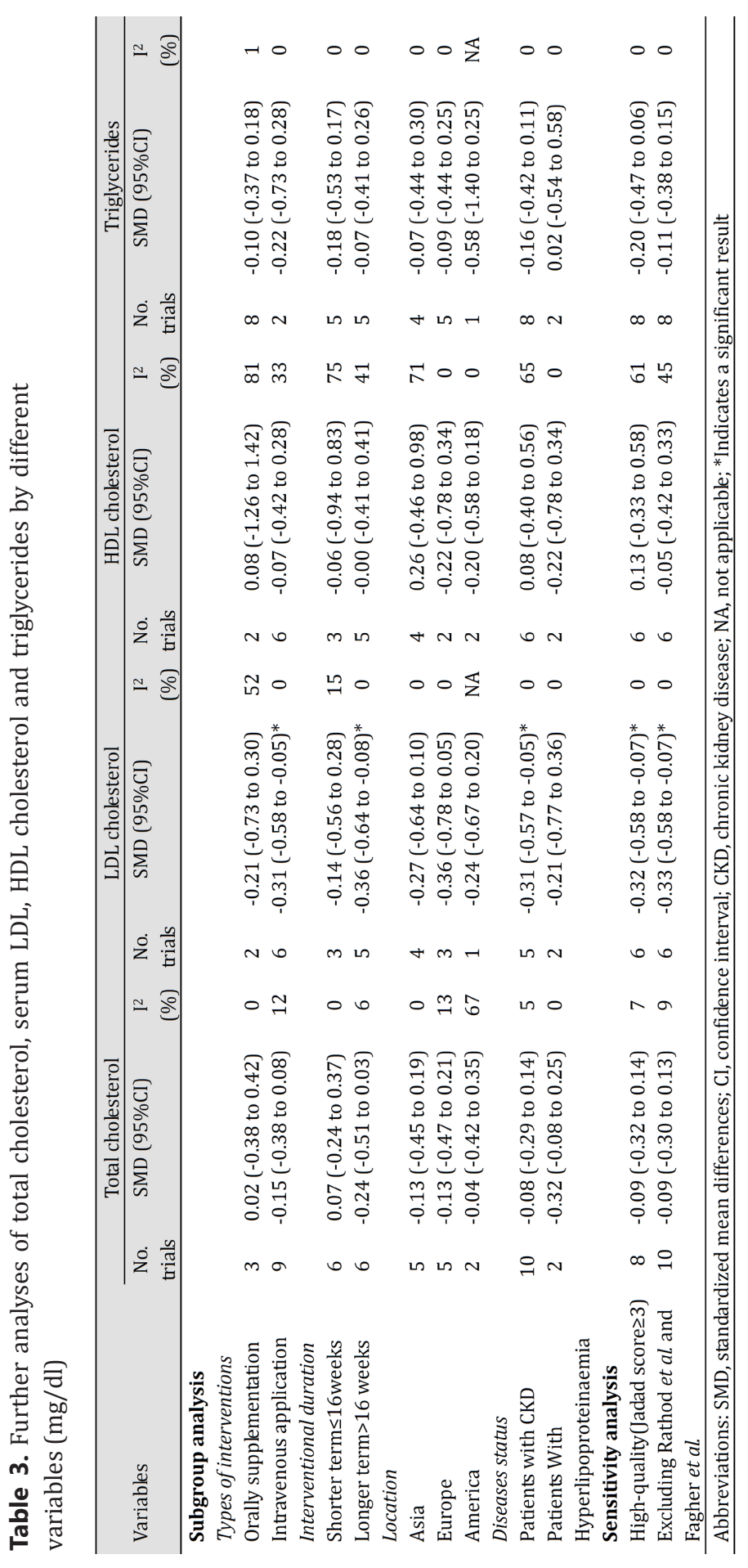

A previous metaanalysis conducted by Hurot et al. [26] implied that there was no evidence of any statistically significant difference in total serum cholesterol, or any of its fractions and serum triglycerides level after L-carnitine supplementation. Following the previous study, our meta-analysis provides a better characterization based on evidence from the effect of L-carnitine supplementation on decreasing serum lipid level in HD patients. Compared with the previous study [26], our study has some advantages: firstly, the sample size is larger than the previous analysis, giving greater power to evaluate this effect. Secondly, based on the previous metaanalysis, further stringent inclusion and exclusion criteria were conducted and other six recent RCTs were included in present analysis [20-25]. Moreover, in order to examine the effect of L-carnitine on various covariants and identify the possible source of heterogeneity within these studies, previously defined subgroup analyses were also performed (the types of interventions, interventional duration, location of subject population, and diseases status of patients). The present meta-analysis suggested that L-carnitine supplementation was not associated with a significant decrease in total serum cholesterol, HDL-cholesterol, VLDL-cholesterol and serum triglycerides level, which is in consistent with the previous meta-analysis [26]. However, it showed that L-carnitine supplementation could significantly decrease the LDL-cholesterol level, which indicates L-carnitine supplementations may be useful for LDL-cholesterol reduction in HD patients. Finally, sensitivity analysis was conducted by omitting any single trial or based on various exclusion criteria, but it did not materially alter the pooled results, which adds robustness to our main point. 


\section{Kidney Blood Pressure Research}

Kidney Blood Press Res 2013;38:31-41

\begin{tabular}{l|l}
\hline DOI: $10.1159 / 000355751$ & C 2014 S. Karger AG, Basel \\
Published onIIne: February 06, 2014 & www.karger.com/kbr
\end{tabular}

Huang/Song/Zhang/Zhang/Zhang/Zhao: L-carnitine Supplementation in HD Patients

There was moderate heterogeneity between studies in the overall analysis of HDLcholesterol, which may be due to different characteristics of the populations, L-carnitine supplementation, and study designs. Our sensitivity analysis suggests that two trials conducted by Rathod et al. [21] and Fagher et al. [16] probably contributed to the heterogeneity. For some patients included in this trial [21] were allowed to use furosemide or iron supplements during the study. A number of studies have demonstrated that use of furosemide may increase the triglycerides and decrease HDL-cholesterol [27, 28]. Iron was reported to promote the synthesis of HDL and accelerate the process of membrane lipid peroxidation in vitro $[29,30]$. In another trial conducted by Fagher et al. [16], we found that some patients enrolled were smokers and this habit remained unchanged during the study. Therefore, these factors may potentially impact on our results and result in the heterogeneity. After omitting both two studies, L-carnitine supplementation was associated with a significant reduce in LDL-cholesterol level (SMD, -0.33; 95\%CI, -0.58 to -0.07), while was not associated with the reduction of TC, HDL and TG levels. Further exclusion of any single article of the included studies, it did not materially alter the overall combined SMD.

The principle finding of our meta-analysis seems to contradict the aforementioned studies on the effects of L-carnitine supplementations on the LDL-cholesterol level. It has been reported that LDL-cholesterol is a risk factor in cardiovascular disease, especially in Coronary Atherosclerotic (AS) [31,32]. Plasma levels of small, dense low-density lipoprotein (LDL) were reported to be increased in chronic kidney disease patients who undergoing HD $[33,34]$. Our meta-analysis revealed that the use of L-carnitine may decrease the LDLcholesterol level in HD patients, which provide a useful information for clinical practice. Moreover, the significant lipid-lowering effects between L-carnitine and LDL-cholesterol appeared to be confined to intravenous application, longer term interventional duration and CKD populations in the subgroup analyses. However, it should be noted that these results are not conclusive and further adequately powered studies are needed. Further studies should pay more attention to this clinical endpoint, so more high-quality randomized clinical trials are warranted.

There were several limitations in our meta-analysis. Firstly, because of the inability to fully adjust for various confounders, the beneficial effect of L-carnitine supplementation on HD patients could be attributed to other healthy habits, such as high fruit and fish consumption. At the same time, some bad habits (eg. smoking and drinking) could also impact on our results. Secondly, owing to different methods used to assess and report L-carnitine supplementation across studies, we failed to evaluate a dose-response relation between L-carnitine supplementation and lipid-lowering effects. Thirdly, although clear inclusion and exclusion criteria were made, significant differences still existed among study design, time on HD, and patient's daily diet. These factors may have a potential impact on our results. Finally, the analysis was only based on published data and unpublished data were not included.

Further studies should focus on the following points. There is a need to standardize a L-carnitine protocol (such as consistency regarding dosage, route, timing, and duration of administration) since great variability exists in the literature. In addition, the synergistic effects of other coexisting substances and L-carnitine on the clinical outcomes of HD patients need to be excluded. Finally, evidence from the current study indicated that L-carnitine are generally considered effective and well tolerated, but future studies should also pay more attention to the side effects of L-carnitine.

\section{Conclusions}

In conclusion, based on current best evidence, our meta-analysis revealed that L-carnitine supplementation was associated with reduction of LDL-cholesterol level, although it was not associated with a significant decrease in total serum cholesterol, HDL-cholesterol, VLDL- 


\section{Kidney \\ Blood Pressure Research}

Huang/Song/Zhang/Zhang/Zhang/Zhao: L-carnitine Supplementation in HD Patients

cholesterol and serum triglycerides level. However, relevant evidence is still limited but is accumulating. Thus, further large-scale, well-designed RCTs are urgently needed.

\section{Conflict of Interests}

The authors have no conflicts of interest to disclose.

\section{Acknowledgements}

We are grateful for useful comments on the manuscript by Dawei Zhang, School of Pharmacy, Guangdong Medical College. This study was funded by the grants from National Natural Science Foundation of China (No. 81173636).

\section{References}

-1 Elliott RW: Demographics of the older adult and chronic kidney disease: a literature review. Nephrol Nurs J 2012;39:491-496; quiz 497.

-2 Kletzmayr J, Horl WH: Iron overload and cardiovascular complications in dialysis patients. Nephrol Dial Transplant 2002;17:S25-29. Cheung AK: Is lipid control necessary in hemodialysis patients? Clin J Am Soc Nephrol 2009;4:S95-101. Borum PR: Carnitine. Annu Rev Nutr 1983;3:233-259.

Carter AL, Abney TO, Lapp DF: Biosynthesis and metabolism of carnitine. J Child Neurol 1995;10:S3-7. Mitwalli AH, Al-Wakeel JS, Alam A, Tarif N, Abu-Aisha H, Rashed M, Al Nahed N: L-carnitine supplementation in hemodialysis patients. Saudi J Kidney Dis Transpl 2005;16:17-22.

7 Irat AM, Aktan F, Ozansoy G: Effects of L-carnitine treatment on oxidant/antioxidant state and vascular reactivity of streptozotocin-diabetic rat aorta. J Pharm Pharmacol 2003;55:1389-1395.

8 Sayed-Ahmed MM, Khattab MM, Gad MZ, Mostafa N: L-carnitine prevents the progression of atherosclerotic lesions in hypercholesterolaemic rabbits. Pharmacol Res 2001;44:235-242.

-9 Liberati A, Altman DG, Tetzlaff J, Mulrow C, Gotzsche PC, Ioannidis JP, Clarke M, Devereaux PJ, Kleijnen J, Moher D: The PRISMA statement for reporting systematic reviews and meta-analyses of studies that evaluate health care interventions: explanation and elaboration. PLoS Med 2009;6:e1000100.

10 Jadad AR, Moore RA, Carroll D, Jenkinson C, Reynolds DJ, Gavaghan DJ, McQuay HJ: Assessing the quality of reports of randomized clinical trials: is blinding necessary? Control Clin Trials 1996;17:1-12.

-11 Kjaergard LL, Villumsen J, Gluud C: Reported methodologic quality and discrepancies between large and small randomized trials in meta-analyses. Ann Intern Med 2001;135:982-989.

12 Higgins JP, Thompson SG, Deeks JJ, Altman DG: Measuring inconsistency in meta-analyses. BMJ 2003;327:557-560.

13 Egger M, Davey Smith G, Schneider M, Minder C: Bias in meta-analysis detected by a simple, graphical test. BMJ 1997;315:629-634.

14 Guarnieri GF, Ranieri F, Toigo G, Vasile A, Ciman M, Rizzoli V, Moracchiello M, Campanacci L: Lipid-lowering effect of carnitine in chronically uremic patients treated with maintenance hemodialysis. Am J Clin Nutr 1980;33:1489-1492.

15 Weschler A, Aviram M, Levin M, Better OS, Brook JG: High dose of L-carnitine increases platelet aggregation and plasma triglyceride levels in uremic patients on hemodialysis. Nephron 1984;38:120-124.

16 Nilsson-Ehle P, Cederblad G, Fagher B, Monti M, Thysell H: Plasma lipoproteins, liver function and glucose metabolism in haemodialysis patients: lack of effect of L-carnitine supplementation. Scand J Clin Lab Invest 1985;45:179-184.

$\checkmark 17$ Yderstraede KB, Pedersen FB, Dragsholt C, Trostmann A, Laier E, Larsen HF: The effect of L-carnitine on lipid metabolism in patients on chronic haemodialysis. Nephrol Dial Transplant 1987;1:238-241. 


\section{Kidney \\ Blood Pressure Research}

\begin{tabular}{l}
\hline Kidney Blood Press Res 2013;38:31-41 \\
\begin{tabular}{l|l}
\hline DOI: 10.1159/000355751 & $\begin{array}{l}\text { C 2014 S. Karger AG, Basel } \\
\text { www.karger.com/kbr }\end{array}$ \\
\hline Published onlIne: February 06, 2014 &
\end{tabular}
\end{tabular}

Huang/Song/Zhang/Zhang/Zhang/Zhao: L-carnitine Supplementation in HD Patients

18 Golper TA, Wolfson M, Ahmad S, Hirschberg R, Kurtin P, Katz LA, Nicora R, Ashbrook D, Kopple JD: Multicenter trial of L-carnitine in maintenance hemodialysis patients. I. Carnitine concentrations and lipid effects. Kidney Int 1990;38:904-911.

$>19$ Labonia WD: L-carnitine effects on anemia in hemodialyzed patients treated with erythropoietin. Am J Kidney Dis 1995;26:757-764.

20 Vaux EC, Taylor DJ, Altmann P, Rajagopalan B, Graham K, Cooper R, Bonomo Y, Styles P: Effects of carnitine supplementation on muscle metabolism by the use of magnetic resonance spectroscopy and near-infrared spectroscopy in end-stage renal disease. Nephron Clin Pract 2004;97:c41-48.

-21 Rathod R, Baig MS, Khandelwal PN, Kulkarni SG, Gade PR, Siddiqui S: Results of a single blind, randomized, placebo-controlled clinical trial to study the effect of intravenous L-carnitine supplementation on healthrelated quality of life in Indian patients on maintenance hemodialysis. Indian J Med Sci 2006;60:143-153.

-22 Duranay M, Akay H, Yilmaz FM, Senes M, Tekeli N, Yucel D: Effects of L-carnitine infusions on inflammatory and nutritional markers in haemodialysis patients. Nephrol Dial Transplant 2006;21:3211-3214.

23 Shakeri A, Tabibi H, Hedayati M: Effects of L-carnitine supplement on serum inflammatory cytokines, C-reactive protein, lipoprotein (a), and oxidative stress in hemodialysis patients with Lp (a) hyperlipoproteinemia. Hemodial Int 2010;14:498-504.

24 Suchitra MM, Ashalatha VL, Sailaja E, Rao AM, Reddy VS, Bitla AR, Sivakumar V, Rao PV: The effect of L-carnitine supplementation on lipid parameters, inflammatory and nutritional markers in maintenance hemodialysis patients. Saudi J Kidney Dis Transpl 2011;22:1155-1159.

-25 Emami Naini A, Moradi M, Mortazavi M, Amini Harandi A, Hadizadeh M, Shirani F, Basir Ghafoori H, Emami Naini P: Effects of Oral L-Carnitine Supplementation on Lipid Profile, Anemia, and Quality of Life in Chronic Renal Disease Patients under Hemodialysis: A Randomized, Double-Blinded, Placebo-Controlled Trial. J Nutr Metab 2012;2012:510483.

-26 Hurot JM, Cucherat M, Haugh M, Fouque D: Effects of L-carnitine supplementation in maintenance hemodialysis patients: a systematic review. J Am Soc Nephrol 2002;13:708-714.

27 Pasternack A, Leino T, Solakivi-Jaakkola T, Huttunen JK, Ehnholm C: Effect of furosemide on the lipid abnormalities in chronic renal failure. Acta Med Scand 1983;214:153-157.

-28 Campbell N, Brant R, Stalts H, Stone J, Mahallati H: Fluctuations in blood lipid levels during furosemide therapy: a randomized, double-blind, placebo-controlled crossover study. Arch Intern Med 1998;158:14611463.

29 Heinecke JW, Rosen H, Chait A: Iron and copper promote modification of low density lipoprotein by human arterial smooth muscle cells in culture. J Clin Invest 1984;74:1890-1894.

30 Macdougall IC, Geisser P: Use of intravenous iron supplementation in chronic kidney disease: an update. Iran J Kidney Dis 2013;7:9-22.

-31 Badimon L, Vilahur G: LDL-cholesterol versus HDL-cholesterol in the atherosclerotic plaque: inflammatory resolution versus thrombotic chaos. Ann N Y Acad Sci 2012;1254:18-32.

-32 Oda H, Yorioka N, Okushin S, Nishida Y, Kushihata S, Ito T, Yamakido M: Remnant-like particle cholesterol may indicate atherogenic risk in patients on chronic hemodialysis. Nephron 1997;76:7-14.

-33 Homma K, Homma Y, Shiina Y, Wakino S, Suzuki M, Fujishima S, Hayashi K, Hori S, Itoh H: Skew of Plasma Low- and High-Density Lipoprotein Distributions to Less Dense Subfractions in Normotriglyceridemic Chronic Kidney Disease Patients on Maintenance Hemodialysis Treatment. Nephron Clin Pract 2013;123:41-45.

34 Shoji T, Ishimura E, Inaba M, Tabata T, Nishizawa Y: Atherogenic lipoproteins in end-stage renal disease. Am J Kidney Dis 2001;38:S30-33. 